\title{
Probing non-spherical dark halos in the Galactic dwarf satellites
}

\author{
Kohei Hayashi and Masashi Chiba \\ Astronomical Institute, Tohoku University, Sendai 980-8578, Japan \\ email: k.hayasi@astr.tohoku.ac.jp \\ email: chiba@astr.tohoku.ac.jp
}

\begin{abstract}
We construct axisymmetric mass models for dwarf spheroidal (dSph) galaxies in the Milky Way to obtain realistic limits on the non-spherical structure of their dark halos. This is motivated by the fact that the observed luminous parts of the dSphs are actually non-spherical and cold dark matter models predict non-spherical virialized dark halos on sub-galactic scales. Applying these models to line-of-sight velocity dispersion profiles along three position angles in six Galactic satellites, we find that the best fitting cases for most of the dSphs yield not spherical but oblate and flattened dark halos. We also find that the mass of the dSphs enclosed within inner $300 \mathrm{pc}$ varies depending on their total luminosities, contrary to the conclusion of previous spherical models. This suggests the importance of considering non-spherical shapes of dark halos in dSph mass models.
\end{abstract}

Keywords. Dwarf spheroidal galaxy, Kinematics and dynamics, Local Group, Dark matter

\section{Mass models}

We construct the following mass models for dSphs. Surface brightness of stars follows an axisymmetric Plummer model. For dark halos, we assume the power-law form $\rho(R, z)=$ $\rho_{0}\left(m / b_{\text {halo }}\right)^{-\alpha}\left[1+\left(m / b_{\text {halo }}\right)^{2}\right]^{-\delta}$, where $m^{2}=R^{2}+z^{2} / Q^{2}, Q$ is an axis ratio, $b_{\text {halo }}$ is a scale length and $\rho_{0}$ is a density normalization. We consider the inclination angle, $i$, of a galaxy, where stellar and halo's principal axes are assumed to be aligned exactly. We then solve the axisymmetric Jeans equations, and determine the halo parameters $\left(Q, b_{\text {halo }}, \rho_{0}, i\right)$ by fitting to the observed line of sight velocity dispersion. We apply these models to six dSphs (Carina, Fornax, Sculptor, Sextans, Draco, LeoI) to obtain their halo parameters. Detailed discussion has been presented in Hayashi \& Chiba (2012).

\section{Flattened dark halos in the dSphs}

Based on $\chi^{2}$ fitting method, we examine the best fit parameters of dark halos for each of six dSphs. We find that shapes of dark matter halos are not spherical (i.e. $Q \neq 1$ ) but significantly flattened $(0.3<Q<0.7)$, both for NFW and cored dark-halo models. In order to compare with spherical mass models, we also estimate the total mass of six dSphs within inner 300 pc (Strigari et al. 2008). It is clear that our axisymmetric mass models provide a different picture on this issue, namely the mass constancy within inner 300 pc as argued by spherical models is not the case.

\section{References}

Hayashi, K., Chiba, M. 2012, ApJ, 755, 145

Strigari, L. E., Bullock, J. S., Kaplinghat, M., Simon, J. D., Geha, M., Willman, B., \& Walker, M. G. 2008, Nature, 454, 1096 